\title{
Robust Stability and Stabilization of a Class of Uncertain Nonlinear Discrete-Time Stochastic Systems with Interval Time-Varying Delays
}

\author{
Shuang Liang and Yali Dong \\ School of Science, Tianjin Polytechnic University, Tianjin 300387, China \\ Correspondence should be addressed to Yali Dong; dongyl@vip.sina.com
}

Received 24 November 2015; Revised 13 February 2016; Accepted 14 February 2016

Academic Editor: Yongji Wang

Copyright @ 2016 S. Liang and Y. Dong. This is an open access article distributed under the Creative Commons Attribution License, which permits unrestricted use, distribution, and reproduction in any medium, provided the original work is properly cited.

\begin{abstract}
This paper deals with the problems of the robust stochastic stability and stabilization for a class of uncertain discrete-time stochastic systems with interval time-varying delays and nonlinear disturbances. By utilizing a new Lyapunov-Krasovskii functional and some well-known inequalities, some new delay-dependent criteria are developed to guarantee the robust stochastic stability of a class of uncertain discrete-time stochastic systems in terms of the linear matrix inequality (LMI). Then based on the state feedback controller, the delay-dependent sufficient conditions of robust stochastic stabilization for a class of uncertain discrete-time stochastic systems with interval time-varying delays are established. The controller gain is designed to ensure the robust stochastic stability of the closed-loop system. Finally, illustrative examples are given to demonstrate the effectiveness of the proposed method.
\end{abstract}

\section{Introduction}

In the past decade, the stability analyses (see, e.g., feedback stabilization for discrete-time nonlinear systems, robustness of exponential stability, and optimal stabilizing compensator) and discrete-time stochastic systems have been extensively studied because of their potential applications (see, e.g., [1-3] and the references therein). On the other hand, time delays, both time-varying and constant, are frequently encountered in various biological, engineering, and economic systems $[4,5]$. The stability analysis and control of time-delay systems have been widely studied during the past years [6-9]. In [6], robust delay-dependent stability and stabilization methods for a class of nonlinear discrete-time systems with timevarying delays were proposed. In [8], the robust stabilization problem for uncertain linear systems with interval timevarying delays was investigated. Some delay-dependent stability criteria were derived based on an improved Wirtinger's inequality.

On the other hand, the research on stochastic systems has aroused much interest in the past few years, because stochastic modeling has come to play an important role in many real systems [10]. In [11], a robust delay-distributiondependent stochastic stability analysis was conducted for a class of discrete-time stochastic delayed neural networks with parameter uncertainties. The robust stability and stabilization of a class of nonlinear discrete stochastic systems were reported in [7]. In [12], the global exponential stability of switched stochastic neural networks with time-varying delays was considered. Authors in [13] studied the robust stability of discrete-time stochastic neural networks with time-varying delays, and the stability analysis problem for stochastic neural networks becomes increasingly significant. In [14], the meansquare exponential stability problem for stochastic discretetime recurrent neural networks with time-varying discrete and distributed delays was investigated. In [15], the delayprobability-distribution-dependent robust stability problem for a class of uncertain stochastic neural networks with timevarying delay was investigated, and some stability criteria were proposed.

In this paper, we contribute to the further development of robust stability and feedback stabilization methods for a class of uncertain nonlinear discrete-time stochastic systems with interval time-varying delays. The parameter uncertainties are time-varying matrices which are norm-bounded, and the unknown nonlinear time-varying perturbations with time-varying delay are quadratically bounded. Comparing 
with $[3,7,8]$, the stochastic nonlinearity and parameter uncertainties and unknown nonlinearities with time-varying delays are considered for discrete-time stochastic systems and therefore the model in this paper may be more general. The main contributions of this paper can be summarized as follows. (1) The system model is comprehensive that covers stochastic nonlinearity, parameter uncertainties, and the unknown nonlinearities that are time-varying perturbations with time-varying delay, thereby better reflecting the reality. (2) An appropriate Lyapunov-Krasovskii functional is constructed to exhibit the delay-dependent dynamics, and delay-dependent robustly stochastic stability analysis is performed to characterize linear matrix inequalities- (LMI-) based conditions under which the discrete-time nonlinear stochastic delay system which does not contain control is robustly stochastically stable. (3) Robust feedback stabilization methods are provided based on state feedback control. The new sufficient conditions are established under which the closed-loop system is robustly stochastically stable and the calculation method of the control gain is given. (4) The result presented in this paper designs a state feedback control law that stabilizes the closed-loop system and is maximally robust with respect to considered nonlinear perturbations. (5) Numerical simulation examples are used to demonstrate the effectiveness and applicability of the obtained results. (6) By introducing some parameters $\epsilon_{1}, \epsilon_{2}, \rho_{1}$, and $\rho_{2}$, our method leads to less conservatism compared with the existing ones.

The remainder of this paper is organized as follows. In Section 2, the problem description and preliminaries are stated and some lemmas and a definition are given. In Section 3, by using Lyapunov-Krasovskii functional, novel LMI sufficient conditions for the robust stochastic stability of a class of uncertain discrete-time stochastic systems with interval time-varying delays and nonlinear disturbances are derived. Furthermore, the robust stochastic stabilizable criteria for uncertain nonlinear discrete-time stochastic delayed systems are presented. In Section 4, two numeric examples are given to illustrate the results. Finally, some conclusions are drawn in Section 5.

Notations. $\mathrm{N}^{+}$denotes the set of all real nonnegative integers and $\mathbb{R}^{n}$ and $\mathbb{R}^{n \times m}$ denote the $n$-dimensional Euclidean space and the set of all $n \times m$ real matrices, respectively. The superscripts $T$ and -1 denote the matrix transposition and matrix inverse, respectively. $\lambda_{\min }(\cdot)$ means the smallest eigenvalue of a matrix. $\mathbb{E}\{\cdot\}$ stands for the mathematical expectation operator with respect to the given probability measure $P$. The asterisk * in a matrix is used to denote term that is induced by symmetry. $I$ is the identity matrix with compatible dimension.

\section{Problem Formulation}

Consider the uncertain nonlinear discrete stochastic system with time-varying delay described by

$$
\begin{aligned}
x(k+1)= & \left(A_{0}+\Delta A_{0}(k)\right) x(k) \\
& +\left(A_{d}+\Delta A_{d}(k)\right) x(k-\tau(k))
\end{aligned}
$$

$$
\begin{aligned}
& +\left(A_{1}+\Delta A_{1}(k)\right) x(k) \xi(k)+f(k, x(k)) \\
& +g(k, x(k-\tau(k)))+(B+\Delta B(k)) u(k),
\end{aligned}
$$

where $x(k) \in \mathbb{R}^{n}$ is the state vector and $k \in I\left[k_{0}, \infty\right)=$ $\left\{k_{0}, k_{0}+1, \ldots\right\} . \xi(k) \in \mathbb{R}$ is a sequence of identically, independently normally distributed random function with

$$
\begin{aligned}
\mathbb{E}[\xi(k)] & =0, \\
\mathbb{E}\left[\xi^{2}(k)\right] & =1, \\
\mathbb{E}[\xi(i) \xi(j)] & =0, \quad(i \neq j),
\end{aligned}
$$

and $u(k) \in \mathbb{R}^{m}$ is the control input. The positive integer $\tau(k)$ denotes the time-varying delay satisfying

$$
\tau_{m} \leq \tau(k) \leq \tau_{M}, \quad \forall k \in N^{+}
$$

where $\tau_{M}$ and $\tau_{m}$ are known positive integers, respectively, and $A_{0} \in \mathbb{R}^{n \times n}, A_{1} \in \mathbb{R}^{n \times n}$, and $B \in \mathbb{R}^{n \times m}$, whereas matrices $\Delta A_{0}(k), \Delta A_{d}(k), \Delta A_{1}(k)$, and $\Delta B(k)$ represent the time-varying parameter uncertainties and are assumed to satisfy the following condition:

$$
\begin{gathered}
\left(\begin{array}{lllll}
\Delta A_{0}(k) & \Delta A_{d}(k) & \Delta A_{1}(k) & \Delta B(k)
\end{array}\right) \\
=D H(k)\left(\begin{array}{lllll}
N_{1} & N_{2} & N_{3} & N_{4}
\end{array}\right)
\end{gathered}
$$

where $D$ and $N_{i}(i=1,2,3,4)$ are known constant matrices and $H(k)$ is the unknown time-varying matrix-valued function satisfying the following condition:

$$
H^{T}(k) H(k) \leq I .
$$

The crucial assumptions about the nonlinear functions $f(k, x(k))$ and $g(k, x(k-\tau(k)))$ are that they are uncertain and satisfy the following quadratic inequalities for all $(k, x) \epsilon$ $I\left[k_{0}, \infty\right) \times \mathbb{R}^{n}:$

$$
\begin{aligned}
& f^{T}(k, x(k)) f(k, x(k)) \leq \alpha^{2} x^{T}(k) F^{T} F x(k), \\
& g^{T}(k, x(k-\tau(k))) g(k, x(k-\tau(k))) \\
& \quad \leq \beta^{2} x^{T}(k-\tau(k)) G^{T} G x(k-\tau(k)) .
\end{aligned}
$$

At the end of this section, we introduce a definition and some lemmas for the development of our results.

Definition 1 (see [7]). System (1) with $u(k) \equiv 0$ is said to be robustly stochastically stable with margins $\alpha$ and $\beta$, if there exists a constant $T\left(x\left(k_{0}\right), \alpha, \beta\right)$ such that

$$
\mathbb{E}\left[\sum_{k=k_{0}}^{\infty} x^{T}(k) x(k) \mid x\left(k_{0}\right)\right] \leq T\left(x\left(k_{0}\right), \alpha, \beta\right) .
$$


Lemma 2 (Schur complements). Given constant matrices $M$, $L$, and $Q$ of appropriate dimensions, where $M=M^{T}$ and $Q>$ 0 , then $M+L^{T} Q L<0$ if and only if

$$
\left(\begin{array}{cc}
M & L^{T} \\
L & -Q^{-1}
\end{array}\right)<0 .
$$

Lemma 3 (see [16]). Let $E, H$, and $F$ be real matrices of appropriate dimensions with $F$ satisfying $F^{T} F \leq I$. Then one has the following inequality. For any scalar $\rho>0$,

$$
E F H+H^{T} F^{T} E^{T} \leq \rho E E^{T}+\rho^{-1} H^{T} H .
$$

\section{Main Results}

The following result presents a sufficient condition of the robustly stochastic stability for system (1).

Theorem 4. For given integers $\tau_{M}>0$ and $\tau_{m}>0$, system (1) with $u(k)=0$ is robustly stochastically stable with margins $\alpha$ and $\beta$, if there exist positive scalars $\rho_{1}, \rho_{2}, \epsilon_{1}$, and $\epsilon_{2}$ and symmetric positive-definite matrices $P$ and $S$ of appropriate dimensions satisfying the following LMI:

$$
\left(\begin{array}{cccccccc}
\Phi_{11} & \rho_{1} N_{1}^{T} N_{2} & A_{0}^{T} P & A_{0}^{T} P & A_{0}^{T} P & A_{1}^{T} P & 0 & 0 \\
* & \Phi_{22} & A_{d}^{T} P & A_{d}^{T} P & A_{d}^{T} P & 0 & 0 & 0 \\
* & * & P-\epsilon_{1} I & P & 0 & 0 & P D & 0 \\
* & * & * & P-\epsilon_{2} I & 0 & 0 & P D & 0 \\
* & * & * & * & -P & 0 & P D & 0 \\
* & * & * & * & 0 & -P & 0 & P D \\
* & * & * & * & * & * & -\rho_{1} I & 0 \\
* & * & * & * & * & * & * & -\rho_{2} I
\end{array}\right)<0,
$$

where

$$
\begin{aligned}
\Phi_{11}= & \left(\tau_{M}-\tau_{m}+1\right) S-P+\rho_{1} N_{1}^{T} N_{1}+\rho_{2} N_{3}^{T} N_{3} \\
& +\epsilon_{1} \alpha^{2} F^{T} F \\
\Phi_{22}= & -S+\rho_{1} N_{2}^{T} N_{2}+\epsilon_{2} \beta^{2} G^{T} G .
\end{aligned}
$$

Proof. Consider the following Lyapunov-Krasovskii functional for system (1):

$$
V(k)=\sum_{i=1}^{3} V_{i}(k)
$$

where

$$
\begin{aligned}
& V_{1}(k)=x^{T}(k) P x(k), \\
& V_{2}(k)=\sum_{i=k-\tau(k)}^{k-1} x^{T}(i) S x(i), \\
& V_{3}(k)=\sum_{j=-\tau_{M}+1}^{-\tau_{m}} \sum_{i=k+j}^{k-1} x^{T}(i) S x(i) .
\end{aligned}
$$

Then, the difference of $V_{i}(k), i=1,2$, along the solution of system (1) is given by

$$
\begin{aligned}
\Delta V_{1}(k)= & x^{T}(k+1) P x(k+1)-x^{T}(k) P x(k), \\
\Delta V_{2}(k)= & \sum_{i=k+1-\tau(k+1)}^{k} x^{T}(i) S x(i)-\sum_{i=k-\tau(k)}^{k-1} x^{T}(i) S x(i) \\
= & \sum_{i=k+1-\tau(k+1)}^{k-\tau_{m}} x^{T}(i) S x(i)+x^{T}(k) S x(k) \\
& -x^{T}(k-\tau(k)) S x(k-\tau(k)) \\
& +\sum_{i=k+1-\tau_{m}}^{k-1} x^{T}(i) S x(i) \\
& -\sum_{i=k+1-\tau(k)}^{k-1} x^{T}(i) S x(i) .
\end{aligned}
$$

Since $\tau_{m} \leq \tau(k) \leq \tau_{M}$, we have

$$
\begin{aligned}
& \sum_{i=k+1-\tau_{m}}^{k-1} x^{T}(i) S x(i)-\sum_{i=k+1-\tau(k)}^{k-1} x^{T}(i) S x(i) \leq 0, \\
& \sum_{i=k+1-\tau(k+1)}^{k-\tau_{m}} x^{T}(i) S x(i) \leq \sum_{i=k+1-\tau_{M}}^{k-\tau_{m}} x^{T}(i) S x(i) .
\end{aligned}
$$


Then we get

$$
\begin{aligned}
\Delta V_{2}(k) \leq & \sum_{i=k+1-\tau_{M}}^{k-\tau_{m}} x^{T}(i) S x(i)+x^{T}(k) S x(k) \\
& -x^{T}(k-\tau(k)) S x(k-\tau(k)) .
\end{aligned}
$$

The difference of $V_{3}(k)$ is given by

$\Delta V_{3}(k)$

$$
\begin{aligned}
= & \sum_{j=-\tau_{M}+1}^{-\tau_{m}} \sum_{i=k+j+1}^{k} x^{T}(i) S x(i) \\
& -\sum_{j=-\tau_{M}+1}^{-\tau_{m}} \sum_{i=k+j}^{k-1} x^{T}(i) S x(i) \\
= & \sum_{j=-\tau_{M}+1}^{-\tau_{m}}\left[x^{T}(k) S x(k)-x^{T}(k+j) S x(k+j)\right] \\
= & \left(\tau_{M}-\tau_{m}\right) x^{T}(k) S x(k) \\
& -\sum_{j=-\tau_{M}+k+1}^{k-\tau_{m}} x^{T}(j) S x(j) .
\end{aligned}
$$

From (17) and (18), we obtain

$$
\begin{aligned}
\Delta V_{2}(k)+\Delta V_{3}(k) \leq & x^{T}(k) S x(k) \\
& -x^{T}(k-\tau(k)) S x(k-\tau(k)) \\
& +\left(\tau_{M}-\tau_{m}\right) x^{T}(k) S x(k) .
\end{aligned}
$$

From (14) and (19), it follows that

$$
\begin{aligned}
\Delta V(k) \leq & x^{T}(k+1) P x(k+1) \\
& +x^{T}(k)\left[\left(\tau_{M}-\tau_{m}+1\right) S-P\right] x(k) \\
& -x^{T}(k-\tau(k)) S x(k-\tau(k)) .
\end{aligned}
$$

According to (6), we have

$$
\begin{aligned}
& \epsilon_{1} \alpha^{2} x^{T}(k) F^{T} F x(k)-\epsilon_{1} f^{T}(k, x(k)) f(k, x(k)) \geq 0, \\
& \epsilon_{2} \beta^{2} x^{T}(k-\tau(k)) G^{T} G x(k-\tau(k)) \\
& \quad-\epsilon_{2} g^{T}(k, x(k-\tau(k))) g(k, x(k-\tau(k))) \geq 0 .
\end{aligned}
$$

From (20) and (21), we get

$\Delta V(k)$

$$
\begin{aligned}
\leq & x^{T}(k+1) P x(k+1) \\
& +x^{T}(k)\left[\left(\tau_{M}-\tau_{m}+1\right) S-P\right] x(k)
\end{aligned}
$$

$$
\begin{aligned}
& -x^{T}(k-\tau(k)) S x(k-\tau(k)) \\
& +\epsilon_{1} \alpha^{2} x^{T}(k) F^{T} F x(k) \\
& -\epsilon_{1} f^{T}(k, x(k)) f(k, x(k)) \\
& +\epsilon_{2} \beta^{2} x^{T}(k-\tau(k)) G^{T} G x(k-\tau(k)) \\
& -\epsilon_{2} g^{T}(k, x(k-\tau(k))) g(k, x(k-\tau(k))) .
\end{aligned}
$$

Let us denote

$$
\begin{aligned}
& \overline{A_{0}}=A_{0}+\Delta A_{0}(k), \\
& \overline{A_{d}}=A_{d}+\Delta A_{d}(k), \\
& \overline{A_{1}}=A_{1}+\Delta A_{1}(k) .
\end{aligned}
$$

Taking the mathematical expectation, we get

$$
\begin{aligned}
& \mathbb{E}\{\Delta V(k)\} \leq \mathbb{E}\left\{\left[x^{T}(k){\overline{A_{0}}}^{T}+x^{T}(k-\tau(k)){\overline{A_{d}}}^{T}\right.\right. \\
& \left.+\xi^{T}(k) x^{T}(k){\overline{A_{1}}}^{T}+f^{T}+g^{T}\right] P\left[\overline{A_{0}} x(k)\right. \\
& \left.+\overline{A_{d}} x(k-\tau(k))+\overline{A_{1}} x(k) \xi(k)+f+g\right] \\
& -x^{T}(k)\left[\left(\tau_{M}-\tau_{m}+1\right) S-P\right] x(k)-x^{T}(k \\
& -\tau(k)) S x(k-\tau(k))+\epsilon_{1} \alpha^{2} x^{T}(k) F^{T} F x(k) \\
& -\epsilon_{1} f^{T}(k, x(k)) f(k, x(k))+\epsilon_{2} \beta^{2} x^{T}(k-\tau(k)) \\
& \cdot G^{T} G x(k-\tau(k))-\epsilon_{2} g^{T}(k, x(k-\tau(k))) \\
& \cdot g(k, x(k-\tau(k)))\} .
\end{aligned}
$$

It is easy to see that

$$
\begin{aligned}
\mathbb{E}\{ & \Delta V(k)\} \leq \mathbb{E}\left\{x ^ { T } ( k ) \left[\epsilon_{1} \alpha^{2} F^{T} F+{\overline{A_{0}}}^{T} P \overline{A_{0}}\right.\right. \\
& \left.+{\overline{A_{1}}}^{T} P \overline{A_{1}}+\left(\tau_{M}-\tau_{m}+1\right) S-P\right] x(k)+x^{T}(k) \\
& \cdot{\overline{A_{0}}}^{T} P \overline{A_{d}} x(k-\tau(k))+x^{T}(k){\overline{A_{0}}}^{T} P f+x^{T}(k) \\
& \cdot{\overline{A_{0}}}^{T} P g+x^{T}(k-\tau(k)){\overline{A_{d}}}^{T} P \overline{A_{0}} x(k)+x^{T}(k \\
& -\tau(k))\left[\epsilon_{2} \beta^{2} G^{T} G+\overline{A_{d}} P \overline{A_{d}}-S\right] x(k-\tau(k)) \\
& +x^{T}(k-\tau(k)) \overline{A_{d}} P f+x^{T}(k-\tau(k)) \overline{A_{d}} P g \\
& +f^{T} P \overline{A_{0}} x(k)+f^{T} P \overline{A_{d}} x(k-\tau(k))+f^{T}[P \\
& \left.-\epsilon_{1} I\right] f+f^{T} P g+g^{T} P \overline{A_{0}} x(k)+g^{T} P \overline{A_{d}} x(k \\
& \left.-\tau(k))+g^{T} P f+g^{T}\left[P-\epsilon_{2} I\right] g\right\} .
\end{aligned}
$$

This reduces to

$$
\mathbb{E}\{\Delta V(k)\} \leq \mathbb{E}\left\{\tilde{x}^{T}(k) \Omega \widetilde{x}(k)\right\},
$$


where

$\tilde{x}(k)$

$=\left(\begin{array}{llll}x^{T}(k) & x^{T}(k-\tau(k)) & f^{T}(k, x(k)) & g^{T}(k, x(k-\tau(k)))\end{array}\right)^{T}$,

$\Omega$

$$
=\left(\begin{array}{cccc}
\Pi & {\overline{A_{0}}}^{T} P \overline{A_{d}} & {\overline{A_{0}}}^{T} P & {\overline{A_{0}}}^{T} P \\
{\overline{A_{d}}}^{T} P \overline{A_{0}} & \epsilon_{2} \beta^{2} G^{T} G+{\overline{A_{d}}}^{T} P \overline{A_{d}}-S & {\overline{A_{d}}}^{T} P & {\overline{A_{d}}}^{T} P \\
P \overline{A_{0}} & P \overline{A_{d}} & P-\epsilon_{1} I & P \\
P \overline{A_{0}} & P \overline{A_{d}} & P & P-\epsilon_{2} I
\end{array}\right),
$$

with $\Pi=\epsilon_{1} \alpha^{2} F^{T} F+{\overline{A_{0}}}^{T} P \overline{A_{0}}+{\overline{A_{1}}}^{T} P \overline{A_{1}}+\left(\tau_{M}-\tau_{m}+1\right) S-P$. Applying Lemma 2, we get that $\Omega<0$ if and only if

$\bar{\Omega}$

$$
=\left(\begin{array}{cccccc}
\bar{\Pi} & 0 & {\overline{A_{0}}}^{T} P & {\overline{A_{0}}}^{T} P & {\overline{A_{0}}}^{T} P & {\overline{A_{1}}}^{T} P \\
* & \epsilon_{2} \beta^{2} G^{T} G-S & {\overline{A_{d}}}^{T} P & {\overline{A_{d}}}^{T} P & {\overline{A_{d}}}^{T} P & 0 \\
* & * & P-\epsilon_{1} I & P & 0 & 0 \\
* & * & * & P-\epsilon_{2} I & 0 & 0 \\
* & * & * & * & -P & 0 \\
* & * & * & * & * & -P
\end{array}\right)
$$

$<0$,

where $\bar{\Pi}=\epsilon_{1} \alpha^{2} F^{T} F+\left(\tau_{M}-\tau_{m}+1\right) S-P$.

We obtain from formula (23) that

$$
\bar{\Omega}=\Phi+\Delta \Phi_{0}(k)+\Delta \Phi_{1}(k)
$$

where

$\Phi$

$$
=\left(\begin{array}{cccccc}
\bar{\Pi} & 0 & A_{0}^{T} P & A_{0}^{T} P & A_{0}^{T} P & A_{1}^{T} P \\
* & \epsilon_{2} \beta^{2} G^{T} G-S & A_{d}^{T} P & A_{d}^{T} P & A_{d}^{T} P & 0 \\
* & * & P-\epsilon_{1} I & P & 0 & 0 \\
* & * & * & P-\epsilon_{2} I & 0 & 0 \\
* & * & * & * & -P & 0 \\
* & * & * & * & * & -P
\end{array}\right),
$$

$\Delta \Phi_{0}(k)$

$$
=\left(\begin{array}{cccccc}
0 & 0 & \Delta A_{0}^{T}(k) P & \Delta A_{0}^{T}(k) P & \Delta A_{0}^{T}(k) P & 0 \\
* & 0 & \Delta A_{d}^{T}(k) P & \Delta A_{d}^{T}(k) P & \Delta A_{d}^{T}(k) P & 0 \\
* & * & 0 & 0 & 0 & 0 \\
* & * & * & 0 & 0 & 0 \\
* & * & * & * & 0 & 0 \\
* & * & * & * & * & 0
\end{array}\right),
$$

$$
\Delta \Phi_{1}(k)=\left(\begin{array}{cccccc}
0 & 0 & 0 & 0 & 0 & \Delta A_{1}^{T}(k) P \\
* & 0 & 0 & 0 & 0 & 0 \\
* & * & 0 & 0 & 0 & 0 \\
* & * & * & 0 & 0 & 0 \\
* & * & * & * & 0 & 0 \\
* & * & * & * & * & 0
\end{array}\right)
$$

From (4) and Lemma 3, we have

$$
\begin{aligned}
& \Delta \Phi_{0}(k)=\left(\begin{array}{cccccc}
0 & 0 & N_{1}^{T} H^{T}(k) D^{T} P & N_{1}^{T} H^{T}(k) D^{T} P & N_{1}^{T} H^{T}(k) D^{T} P & 0 \\
* & 0 & N_{2}^{T} H^{T}(k) D^{T} P & N_{2}^{T} H^{T}(k) D^{T} P & N_{2}^{T} H^{T}(k) D^{T} P & 0 \\
* & * & 0 & 0 & 0 & 0 \\
* & * & * & 0 & 0 & 0 \\
* & * & * & * & 0 & 0 \\
* & * & * & * & * & 0
\end{array}\right) \\
& =\left(\begin{array}{c}
0 \\
0 \\
P D \\
P D \\
P D \\
0
\end{array}\right) H(k)\left(\begin{array}{llllll}
N_{1} & N_{2} & 0 & 0 & 0 & 0
\end{array}\right)+\left(\begin{array}{c}
N_{1}^{T} \\
N_{2}^{T} \\
0 \\
0 \\
0 \\
0
\end{array}\right) H^{T}(k)\left(\begin{array}{lllllll}
0 & 0 & D^{T} P & D^{T} P & D^{T} P & 0
\end{array}\right)
\end{aligned}
$$




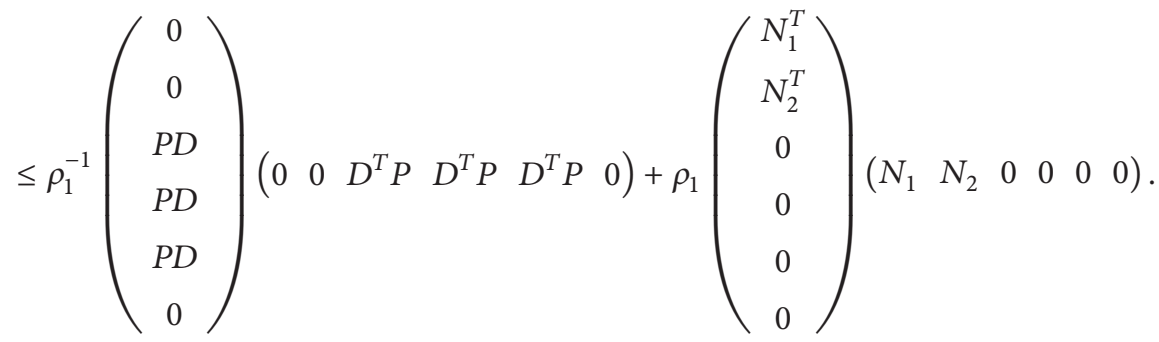

Similarly, it is not difficult to verify that

$$
\Delta \Phi_{1}(k) \leq\left(\begin{array}{cccccc}
0 & 0 & 0 & 0 & 0 & 0 \\
* & 0 & 0 & 0 & 0 & 0 \\
* & * & 0 & 0 & 0 & 0 \\
* & * & * & 0 & 0 & 0 \\
* & * & * & * & 0 & 0 \\
* & * & * & * & * & \rho_{2}^{-1} P D D^{T} P
\end{array}\right)
$$

$$
+\left(\begin{array}{cccccc}
\rho_{2} N_{3}^{T} N_{3} & 0 & 0 & 0 & 0 & 0 \\
* & 0 & 0 & 0 & 0 & 0 \\
* & * & 0 & 0 & 0 & 0 \\
* & * & * & 0 & 0 & 0 \\
* & * & * & * & 0 & 0 \\
* & * & * & * & * & 0
\end{array}\right)
$$

From (30), (32), and (33), we get

$$
\bar{\Omega} \leq\left(\begin{array}{cccccc}
\Pi_{11} & \rho_{1} N_{1}^{T} N_{2} & A_{0}^{T} P & A_{0}^{T} P & A_{0}^{T} P & A_{1}^{T} P \\
* & \Pi_{22} & A_{d}^{T} P & A_{d}^{T} P & A_{d}^{T} P & 0 \\
* & * & \Pi_{33} & P+\rho_{1}^{-1} P D D^{T} P & \rho_{1}^{-1} P D D^{T} P & 0 \\
* & * & * & \Pi_{44} & \rho_{1}^{-1} P D D^{T} P & 0 \\
* & * & * & * & \Pi_{55} & 0 \\
* & * & * & * & * & \Pi_{66}
\end{array}\right),
$$

where

$$
\begin{aligned}
\Pi_{11}= & \epsilon_{1} \alpha^{2} F^{T} F+\left(\tau_{M}-\tau_{m}+1\right) S-P+\rho_{1} N_{1}^{T} N_{1} \\
& +\rho_{2} N_{3}^{T} N_{3}, \\
\Pi_{22}= & \epsilon_{2} \beta^{2} G^{T} G-S+\rho_{1} N_{2}^{T} N_{2}, \\
\Pi_{33}= & P-\epsilon_{1} I+\rho_{1}^{-1} P D D^{T} P \\
\Pi_{44}= & P-\epsilon_{2} I+\rho_{1}^{-1} P D D^{T} P, \\
\Pi_{55}= & -P+\rho_{1}^{-1} P D D^{T} P \\
\Pi_{66}= & -P+\rho_{2}^{-1} P D D^{T} P .
\end{aligned}
$$

From (10), we get that $\bar{\Omega}<0$, which implies $\Omega<0$. Hence, we have

$$
\sum_{i=1}^{3} \Delta V_{i}(k) \leq-\lambda_{\min }(-\Omega) \tilde{x}^{T}(k) \tilde{x}(k)
$$

Taking expected value and summing up both sides of the above equation for $T \geq k_{0}$, we have

$$
\begin{aligned}
\mathbb{E} & {[V(T, x(T))]-\mathbb{E}\left[V\left(k_{0}, x\left(k_{0}\right)\right)\right] } \\
& =\mathbb{E}\left[\sum_{k=k_{0}}^{T-1} \Delta V(k, x(k)) \mid x\left(k_{0}\right)\right] \\
& \leq-\lambda_{\min }(-\Omega) \mathbb{E}\left[\sum_{k=k_{0}}^{T} \tilde{x}^{T}(k) \tilde{x}(k) \mid x\left(k_{0}\right)\right] .
\end{aligned}
$$

Thus,

$$
\begin{aligned}
& \lambda_{\min }(-\Omega) \mathbb{E}\left[\sum_{k=k_{0}}^{T} \tilde{x}^{T}(k) \tilde{x}(k) \mid x\left(k_{0}\right)\right] \\
& \leq \mathbb{E}\left[V\left(k_{0}, x\left(k_{0}\right)\right)\right]-\mathbb{E}[V(T, x(T))] \\
& \leq \mathbb{E}\left[V\left(k_{0}, x\left(k_{0}\right)\right)\right] .
\end{aligned}
$$


We get

$$
\mathbb{E}\left[\sum_{k=k_{0}}^{T} \tilde{x}^{T}(k) \tilde{x}(k) \mid x\left(k_{0}\right)\right] \leq \frac{\mathbb{E}\left[V\left(k_{0}, x\left(k_{0}\right)\right)\right]}{\lambda_{\min }(-\Omega)} .
$$

Obviously, $\|x\| \leq\|\widetilde{x}\|$ and this leads to

$$
\mathbb{E}\left[\sum_{k=k_{0}}^{T} x^{T}(k) x(k) \mid x\left(k_{0}\right)\right] \leq \frac{\mathbb{E}\left[V\left(k_{0}, x\left(k_{0}\right)\right)\right]}{\lambda_{\min }(-\Omega)},
$$

which leads to the robust stochastic stability of (1) with $u(k)=$ 0 with margins $\alpha$ and $\beta$. This completes the proof of the theorem.

Remark 5. In [7], the stochastic stability analysis problem had been studied for discrete-time system with stochastic disturbance. But the time-delay and parameter uncertainties and unknown nonlinearities with time-varying delays were not considered in [7]. In this paper, we consider the timedelay and parameter uncertainties and the unknown nonlinear time-varying perturbations with time-varying delay. Comparing with [7], the model that is given by uncertain nonlinear discrete-time stochastic system (1) is a more general one.

Remark 6. In this paper, scalars $\epsilon_{1}, \epsilon_{2}, \rho_{1}$, and $\rho_{2}$ are introduced with the aim to obtain a tractable matrix condition, while the conservatism does not increase much. Compared to [7], by choosing these scalars appropriately, the conservatism can be further reduced.

We have reformulated this theorem as an optimization problem which is given below as a separated theorem.

Theorem 7. Let $\gamma_{0}$ and $\gamma_{1}$ be the optimal solutions of the following optimization problem:

$$
\begin{array}{ll}
\operatorname{maximize} & \gamma, \tilde{\gamma} \\
\text { subject to } & P>0, \\
& S>0, \\
& \rho_{1}>0, \\
& \rho_{2}>0,
\end{array}
$$

for some $\epsilon_{1}>0, \epsilon_{2}>0$,

$$
\left(\begin{array}{cccccccc}
\Phi_{11} & \rho_{1} N_{1}^{T} N_{2} & A_{0}^{T} P & A_{0}^{T} P & A_{0}^{T} P & A_{1}^{T} P & 0 & 0 \\
* & \Phi_{22} & A_{d}^{T} P & A_{d}^{T} P & A_{d}^{T} P & 0 & 0 & 0 \\
* & * & P-\epsilon_{1} I & P & 0 & 0 & P D & 0 \\
* & * & * & P-\epsilon_{2} I & 0 & 0 & P D & 0 \\
* & * & * & * & -P & 0 & P D & 0 \\
* & * & * & * & 0 & -P & 0 & P D \\
* & * & * & * & * & * & -\rho_{1} I & 0 \\
* & * & * & * & * & * & * & -\rho_{2} I
\end{array}\right)<0
$$

where

$$
\begin{aligned}
\Phi_{11}= & \left(\tau_{M}-\tau_{m}+1\right) S-P+\rho_{1} N_{1}^{T} N_{1}+\rho_{2} N_{3}^{T} N_{3} \\
& +\gamma F^{T} F, \\
\Phi_{22}= & -S+\rho_{1} N_{2}^{T} N_{2}+\tilde{\gamma} G^{T} G .
\end{aligned}
$$

Then, for any $0<\gamma \leq \gamma_{0}$ and $0<\tilde{\gamma} \leq \gamma_{1}$, system (1) with $u(k)=$ 0 is robustly stochastically stable with margins $\alpha=\sqrt{\gamma / \epsilon_{1}}$ and $\beta=\sqrt{\widetilde{\gamma} / \epsilon_{2}}$.

We now consider the problem of robustly stochastic stability of system (1).

Theorem 8. System (1) is robustly stochastically stabilizable with margins $\alpha$ and $\beta$ under the controller $u(k)=K x(k)$ with $K=Y X^{-1}$, if there exist positive scalars $\rho_{1}, \rho_{2}$, and $\rho_{3}$, symmetric positive-definite matrices $X$ and $Q$, and any matrix $Y$ of appropriate dimensions satisfying the following LMI: 


$$
\left(\begin{array}{cccccccccccc}
-X & 0 & J^{T} & J^{T} & J^{T} & X A_{1}^{T} & X & X N_{1}^{T} & X N_{3}^{T} & Y^{T} N_{4}^{T} & X F^{T} & 0 \\
* & \Xi_{22} & X A_{d}^{T} & X A_{d}^{T} & X A_{d}^{T} & 0 & 0 & X N_{2}^{T} & 0 & 0 & 0 & X G^{T} \\
* & * & \Xi_{33} & \Xi_{34} & \Xi_{35} & 0 & 0 & 0 & 0 & 0 & 0 & 0 \\
* & * & * & \Xi_{44} & \Xi_{45} & 0 & 0 & 0 & 0 & 0 & 0 & 0 \\
* & * & * & * & \Xi_{55} & 0 & 0 & 0 & 0 & 0 & 0 & 0 \\
* & * & * & * & * & \Xi_{66} & 0 & 0 & 0 & 0 & 0 & 0 \\
* & * & * & * & * & * & \Xi_{77} & 0 & 0 & 0 & 0 & 0 \\
* & * & * & * & * & * & * & -\rho_{1} I & 0 & 0 & 0 & 0 \\
* & * & * & * & * & * & * & * & -\rho_{2} I & 0 & 0 & 0 \\
* & * & * & * & * & * & * & * & * & -\rho_{3} I & 0 & 0 \\
* & * & * & * & * & * & * & * & * & * & -\alpha^{-2} I & 0 \\
* & * & * & * & * & * & * & * & * & * & * & -\beta^{-2} I
\end{array}\right)<
$$

where

$$
\begin{aligned}
& \Xi_{22}=Q-2 X, \\
& \Xi_{33}=I-X+\rho_{1} D D^{T}+\rho_{3} D D^{T}, \\
& \Xi_{34}=X+\rho_{1} D D^{T}+\rho_{3} D D^{T}, \\
& \Xi_{35}=\rho_{1} D D^{T}+\rho_{3} D D^{T}, \\
& \Xi_{44}=I-X+\rho_{1} D D^{T}+\rho_{3} D D^{T}, \\
& \Xi_{45}=\rho_{1} D D^{T}+\rho_{3} D D^{T}, \\
& \Xi_{55}=-X+\rho_{1} D D^{T}+\rho_{3} D D^{T}, \\
& \Xi_{66}=-X+\rho_{2} D D^{T}, \\
& \Xi_{77}=-\left(\tau_{M}-\tau_{m}+1\right)^{-1} Q, \\
& J=A_{0} X+B Y .
\end{aligned}
$$

Proof. Substituting $u(k)=K x(k)$ into (1) yields the dynamics of the closed-loop system described by

$$
\begin{gathered}
x(k+1)=\left[A_{0}+\Delta A_{0}(k)+(B+\Delta B(k)) K\right] x(k) \\
+\left(A_{d}+\Delta A_{d}(k)\right) x(k-\tau(k))
\end{gathered}
$$

$$
\begin{aligned}
& +\left(A_{1}+\Delta A_{1}(k)\right) x(k) \xi(k) \\
& +f(k, x(k))+g(k, x(k-\tau(k))) \\
= & {\left[\overline{A_{0}}+\bar{B} K\right] x(k)+\overline{A_{d}} x\left(k-\tau_{k}\right) } \\
& +\overline{A_{1}} x(k) \xi(k)+f(k, x(k)) \\
& +g(k, x(k-\tau(k))) .
\end{aligned}
$$

Denote $\widehat{A}=\overline{A_{0}}+\bar{B} K$.

Similar to the proof of Theorem 4, we get that the closed-loop system (45) is stochastically stable if there exist symmetric positive-definite matrices $P$ and $S$ satisfying the following LMI:

$\Psi$

$$
=\left(\begin{array}{cccccc}
\Pi_{11} & 0 & {\widehat{A_{0}}}^{T} P & {\widehat{A_{0}}}^{T} P & {\widehat{A_{0}}}^{T} P & {\overline{A_{1}}}^{T} P \\
* & \beta^{2} G^{T} G-S & {\overline{A_{d}}}^{T} P & {\overline{A_{d}}}^{T} P & {\overline{A_{d}}}^{T} P & 0 \\
* & * & P-I & P & 0 & 0 \\
* & * & * & P-I & 0 & 0 \\
* & * & * & * & -P & 0 \\
* & * & * & * & * & -P
\end{array}\right)
$$
where $\Pi_{11}=\alpha^{2} F^{T} F+\left(\tau_{M}-\tau_{m}+1\right) S-P$. 


$$
\Psi \leq\left(\begin{array}{cccccc}
W_{11} & \rho_{1}^{-1} N_{1}^{T} N_{2} & A_{0}^{T} P+K^{T} B^{T} P & A_{0}^{T} P+K^{T} B^{T} P & A_{0}^{T} P+K^{T} B^{T} P & A_{1}^{T} P \\
* & W_{22} & A_{d}^{T} P & A_{d}^{T} P & A_{d}^{T} P & 0 \\
* & * & W_{33} & W_{34} & W_{35} & 0 \\
* & * & * & W_{44} & W_{45} & 0 \\
* & * & * & * & W_{55} & 0 \\
* & * & * & * & * & W_{66}
\end{array}\right)
$$

where

$$
\begin{aligned}
W_{11}= & \alpha^{2} F^{T} F+\left(\tau_{M}-\tau_{m}+1\right) S-P+\rho_{1}^{-1} N_{1}^{T} N_{1} \\
& +\rho_{2}^{-1} N_{3}^{T} N_{3}+\rho_{3}^{-1} K^{T} N_{4}^{T} N_{4} K \\
W_{22}= & \beta^{2} G^{T} G-S+\rho_{1}^{-1} N_{2}^{T} N_{2} \\
W_{33}= & P-I+\rho_{1} P D D^{T} P+\rho_{3} P D D^{T} P \\
W_{34}= & P+\rho_{1} P D D^{T} P+\rho_{3} P D D^{T} P \\
W_{35}= & \rho_{1} P D D^{T} P+\rho_{3} P D D^{T} P
\end{aligned}
$$

$$
\begin{aligned}
& W_{44}=P-I+\rho_{1} P D D^{T} P+\rho_{3} P D D^{T} P, \\
& W_{45}=\rho_{1} P D D^{T} P+\rho_{3} P D D^{T} P, \\
& W_{55}=-P+\rho_{1} P D D^{T} P+\rho_{3} P D D^{T} P, \\
& W_{66}=-P+\rho_{2} P D D^{T} P .
\end{aligned}
$$

Let $P^{-1}=X, Q=S^{-1}$, and pre- and postmultiplying (47) by $\operatorname{diag}\{X, X, X, X, X, X\}$ yield

$$
\begin{aligned}
\bar{\Psi} & =\operatorname{diag}\{X, X, X, X, X, X\} \Psi \operatorname{diag}\{X, X, X, X, X, X\} \\
& =\left(\begin{array}{cccccc}
W_{11} & \rho_{1}^{-1} X N_{1}^{T} N_{2} X & X A_{0}^{T}+X K^{T} B^{T} & X A_{0}^{T}+X K^{T} B^{T} & X A_{0}^{T}+X K^{T} B^{T} & X A_{1}^{T} \\
* & W_{22} & X A_{d}^{T} & X A_{d}^{T} & X A_{d}^{T} & 0 \\
* & * & W_{33} & W_{34} & W_{35} & 0 \\
* & * & * & W_{44} & W_{45} & 0 \\
* & * & * & * & W_{55} & 0 \\
* & * & * & * & * & W_{66}
\end{array}\right),
\end{aligned}
$$

where

$$
\begin{aligned}
W_{11}= & \alpha^{2} X F^{T} F X+\left(\tau_{M}-\tau_{m}+1\right) X S X-X \\
& +\rho_{1}^{-1} X N_{1}^{T} N_{1} X+\rho_{2}^{-1} X N_{3}^{T} N_{3} X \\
& +\rho_{3}^{-1} X K^{T} N_{4}^{T} N_{4} K X, \\
W_{22}= & \beta^{2} X G^{T} G X-X S X+\rho_{1}^{-1} X N_{2}^{T} N_{2} X, \\
W_{33}= & X-X^{2}+\rho_{1} D D^{T}+\rho_{3} D D^{T}, \\
W_{34}= & X+\rho_{1} D D^{T}+\rho_{3} D D^{T}, \\
W_{35}= & \rho_{1} D D^{T}+\rho_{3} D D^{T}, \\
W_{44}= & X-X^{2}+\rho_{1} D D^{T}+\rho_{3} D D^{T},
\end{aligned}
$$

$$
\begin{aligned}
& W_{45}=\rho_{1} D D^{T}+\rho_{3} D D^{T}, \\
& W_{55}=-X+\rho_{1} D D^{T}+\rho_{3} D D^{T}, \\
& W_{66}=-X+\rho_{2} D D^{T} .
\end{aligned}
$$

Using the well-known relationships

$$
\begin{aligned}
X+X^{-1} & \geq 2 I, \\
W^{T}-2 X & \geq X^{T} W^{-1} X,
\end{aligned}
$$

we can get

$$
\begin{gathered}
X-X^{2} \leq I-X, \\
X^{T} W^{-1} X \geq X+X^{T}-W .
\end{gathered}
$$


Using (52) and the gain matrix $K=Y X^{-1}$, we obtain

$$
\bar{\Psi} \leq\left(\begin{array}{cccccc}
W_{11} & \rho_{1}^{-1} X N_{1}^{T} N_{2} X & X A_{0}^{T}+Y^{T} B^{T} & X A_{0}^{T}+Y^{T} B^{T} & X A_{0}^{T}+Y^{T} B^{T} & X A_{1}^{T} \\
* & W_{22} & X A_{d}^{T} & X A_{d}^{T} & X A_{d}^{T} & 0 \\
* & * & W_{33} & W_{34} & W_{35} & 0 \\
* & * & * & W_{44} & W_{45} & 0 \\
* & * & * & * & W_{55} & 0 \\
* & * & * & * & * & W_{66}
\end{array}\right) \text {, }
$$

where

$$
\begin{aligned}
& W_{11}= \alpha^{2} X F^{T} F X+\left(\tau_{M}-\tau_{m}+1\right) X S X-X \\
&+\rho_{1}^{-1} X N_{1}^{T} N_{1} X+\rho_{2}^{-1} X N_{3}^{T} N_{3} X \\
&+\rho_{3}^{-1} Y^{T} N_{4}^{T} N_{4} Y, \\
& W_{22}= \beta^{2} X G^{T} G X+S^{-1}-2 X+\rho_{1}^{-1} X N_{2}^{T} N_{2} X, \\
& W_{33}= I-X+\rho_{1} D D^{T}+\rho_{3} D D^{T}, \\
& W_{34}=X+\rho_{1} D D^{T}+\rho_{3} D D^{T}, \\
& W_{35}=\rho_{1} D D^{T}+\rho_{3} D D^{T}, \\
& W_{44}=I-X+\rho_{1} D D^{T}+\rho_{3} D D^{T}, \\
& W_{45}=\rho_{1} D D^{T}+\rho_{3} D D^{T},
\end{aligned}
$$

$$
\begin{aligned}
& W_{55}=-X+\rho_{1} D D^{T}+\rho_{3} D D^{T}, \\
& W_{66}=-X+\rho_{2} D D^{T} .
\end{aligned}
$$

We have known that $\Psi<0$ is equivalent to $\bar{\Psi}<0$. By Lemma 2, we get LMI (43) implying that $\bar{\Psi}<0$, which concludes the proof of the theorem.

Remark 9. The proposed feedback controller can ensure stochastic stability of the closed-loop system in Theorem 8 . If $\alpha$ and $\beta$ are given, the feasibility problem of LMI can be solved to get a suitable stabilization controller gain.

We have reformulated this theorem as an optimization problem which is given below as a separated theorem.

Theorem 10. Let $\gamma_{0}$ and $\gamma_{1}$ be the optimal solutions of the following optimization problem:

$$
\begin{array}{cl}
\text { minimize } & \gamma, \tilde{\gamma} \\
\text { subject to } & X>0, \\
& Q>0,
\end{array}
$$


with

$$
\begin{aligned}
& \Xi_{22}=Q-2 X, \\
& \Xi_{33}=I-X+\rho_{1} D D^{T}+\rho_{3} D D^{T}, \\
& \Xi_{34}=X+\rho_{1} D D^{T}+\rho_{3} D D^{T}, \\
& \Xi_{35}=\rho_{1} D D^{T}+\rho_{3} D D^{T}, \\
& \Xi_{44}=I-X+\rho_{1} D D^{T}+\rho_{3} D D^{T}, \\
& \Xi_{45}=\rho_{1} D D^{T}+\rho_{3} D D^{T}, \\
& \Xi_{55}=-X+\rho_{1} D D^{T}+\rho_{3} D D^{T}, \\
& \Xi_{66}=-X+\rho_{2} D D^{T}, \\
& \Xi_{77}=-\left(\tau_{M}-\tau_{m}+1\right)^{-1} Q, \\
& J=A_{0} X+B Y .
\end{aligned}
$$

Then, for any $\gamma \geq \gamma_{0}$ and $\tilde{\gamma} \geq \gamma_{1}$, system (1) with $u(k)=K x(k)$, where $K=Y X^{-1}$, is robustly stochastically stable with margins $\alpha=1 / \sqrt{\gamma}$ and $\beta=1 / \sqrt{\bar{\gamma}}$.

Remark 11. Unlike robust control results available in the literature $[17,18]$, the result presented in this paper designs a linear control law that stabilizes the closed-loop system and is maximally robust with respect to considered nonlinear perturbations.

Remark 12. In [19], by using a linear controller, delaydependent sufficient conditions of stabilization for a class of nonlinear discrete-time systems with varying time delay were given. However, the system in [19] did not involve stochastic disturbance. In [7], authors considered the robust state feedback stability and stabilization of nonlinear discretetime stochastic system, but the stochastic system in [7] did not include time delay. Compared with $[7,19]$, the results obtained in this paper have a greater range of applications.

Remark 13. In this paper, we use the linear state feedback control law which has many applications in stochastic stability analysis and control synthesizing. For example, in [20], for the robust stabilization problem, a linear state feedback controller was designed, which ensured that the closed-loop system was robustly stochastically stable with maximal decay rate. In [7], a linear state feedback controller was used to explore the stabilization of a class of nonlinear discrete-time stochastic systems. In [21], asymptotic stabilization of a discrete-time switched stochastic system was investigated based on a linear state feedback controller.

\section{Numerical Examples}

In this section, two numerical examples are provided to illustrate the usefulness of the proposed criteria.
Example 1. Consider system (1) with $u(k)=0$ and the following parameters:

$$
\begin{aligned}
& A_{0}=\left(\begin{array}{ll}
0.3 & 0.2 \\
0.2 & 0.3
\end{array}\right), \\
& A_{d}=\left(\begin{array}{cc}
0.5 & 0.16 \\
0.16 & 0.4
\end{array}\right) \text {, } \\
& A_{1}=\left(\begin{array}{cc}
-0.12 & 0.08 \\
0.08 & -0.1
\end{array}\right) \text {, } \\
& F=G=\left(\begin{array}{ll}
1 & 0 \\
0 & 1
\end{array}\right) \text {, } \\
& D=\left(\begin{array}{cc}
-0.3 & 0.1 \\
0.1 & -0.3
\end{array}\right) \text {, } \\
& N_{1}=\left(\begin{array}{ll}
0.1 & 0.1 \\
0.1 & 0.1
\end{array}\right) \text {, } \\
& N_{2}=\left(\begin{array}{cc}
-0.1 & 0.1 \\
0.1 & 0.1
\end{array}\right) \text {, } \\
& N_{3}=\left(\begin{array}{cc}
-0.1 & 0.1 \\
0.1 & -0.1
\end{array}\right) \text {, } \\
& \tau(k)=2+\sin \frac{k \pi}{2}, \\
& \tau_{M}=3 \text {, } \\
& \tau_{m}=1 \text {, } \\
& \epsilon_{1}=\epsilon_{2}=1 \text {, } \\
& \alpha=0.8 \text {, } \\
& \beta=1.5 \text {. }
\end{aligned}
$$

By using Matlab LMI Toolbox, we solve LMI (10) and obtain the feasible solutions as follows:

$$
\begin{aligned}
P & =\left(\begin{array}{cc}
0.2115 & -0.1316 \\
-0.1316 & 0.2679
\end{array}\right), \\
S & =\left(\begin{array}{cc}
0.0290 & -0.0322 \\
-0.0322 & 0.0466
\end{array}\right), \\
\rho_{1} & =0.1018, \\
\rho_{2} & =0.5570 .
\end{aligned}
$$

The simulation of the state response of $x(k)$ under initial condition $x(0)=(-1,1)^{T}$ is given in Figure 1 . 


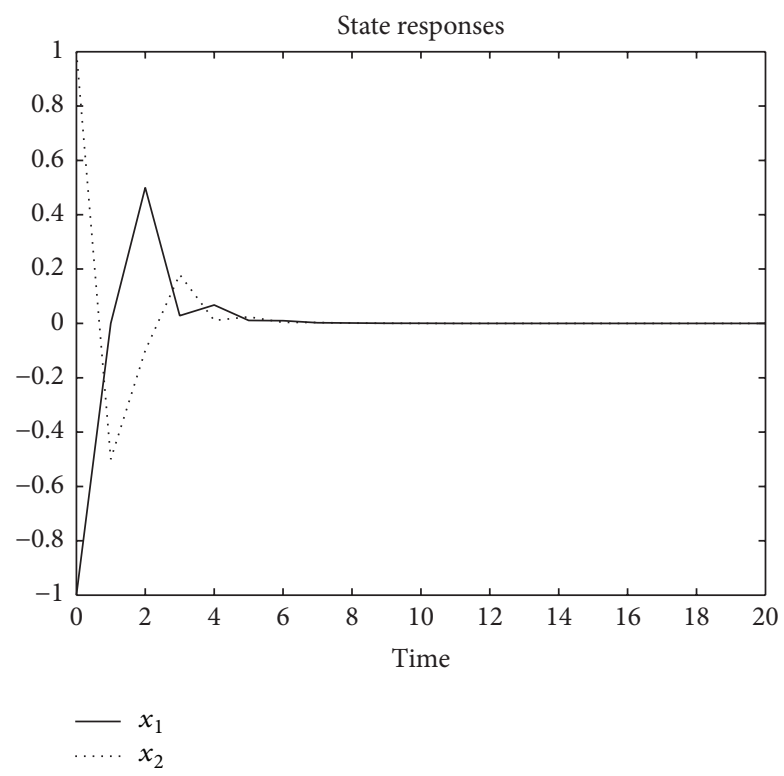

FIGURE 1: State trajectories of the open-loop system.

Example 2. We consider the uncertain nonlinear discrete stochastic system (1) with the following parameters:

$$
\begin{aligned}
& A_{0}=\left(\begin{array}{ll}
0.5 & 2 \\
1.8 & 1
\end{array}\right), \\
& A_{d}=\left(\begin{array}{cc}
0.1 & -0.4 \\
-0.5 & 0.5
\end{array}\right) \text {, } \\
& A_{1}=\left(\begin{array}{cc}
-0.12 & 0.08 \\
0.08 & -0.1
\end{array}\right) \text {, } \\
& B=\left(\begin{array}{cc}
0.2 & -0.3 \\
-0.3 & 0.2
\end{array}\right) \text {, } \\
& D=\left(\begin{array}{cc}
0.1 & 0.1 \\
0.1 & -0.03
\end{array}\right) \text {, } \\
& N_{1}=\left(\begin{array}{ll}
0.1 & 0.2 \\
0.2 & 0.1
\end{array}\right) \text {, } \\
& N_{2}=\left(\begin{array}{ll}
0.1 & 0.1 \\
0.1 & 0.1
\end{array}\right) \text {, } \\
& N_{3}=\left(\begin{array}{ll}
0.1 & 0.1 \\
0.1 & 0.1
\end{array}\right) \text {, } \\
& N_{4}=\left(\begin{array}{ll}
0.1 & 0.1 \\
0.1 & 0.1
\end{array}\right) \text {, } \\
& F=G=I=\left(\begin{array}{ll}
1 & 0 \\
0 & 1
\end{array}\right) \text {, } \\
& \tau(k)=2+\sin \frac{k \pi}{2} .
\end{aligned}
$$

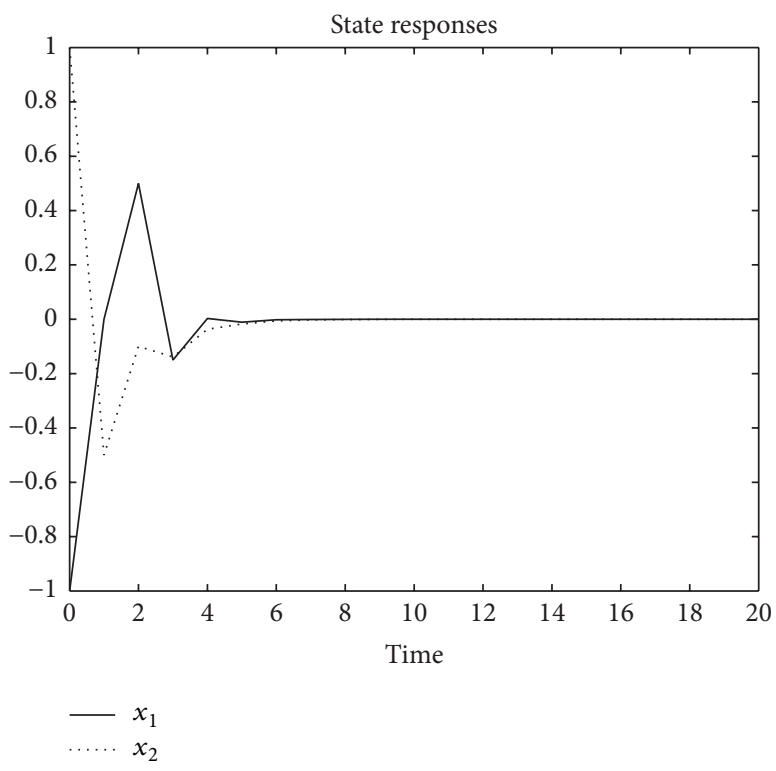

FIGURE 2: State trajectories of the closed-loop system.

Given that $\alpha=1.25, \beta=0.8, \tau_{M}=3$, and $\tau_{m}=1$, then the solution of LMI (40) is

$$
\begin{aligned}
X & =\left(\begin{array}{cc}
0.1730 & -0.0646 \\
-0.0646 & 0.1673
\end{array}\right), \\
Q & =\left(\begin{array}{cc}
0.1183 & -0.0670 \\
-0.0670 & 0.1003
\end{array}\right), \\
Y & =\left(\begin{array}{ll}
1.1881 & 0.6066 \\
0.6066 & 1.1076
\end{array}\right), \\
\rho_{1} & =0.1337, \\
\rho_{2} & =0.2596, \\
\rho_{3} & =0.1738 .
\end{aligned}
$$

By the formula $K=Y X^{-1}$, we get the controller gain

$$
K=\left(\begin{array}{ll}
9.6041 & 7.3341 \\
6.9836 & 9.3173
\end{array}\right) \text {. }
$$

Figure 2 shows the simulation results for states $x_{1}(k)$ and $x_{2}(k)$ under initial condition $x(0)=(-1,1)^{T}$. Simulation results demonstrate that our proposed design is very effective.

\section{Conclusions}

In this paper, we have investigated the robust stochastic stability and stabilization for a class of uncertain nonlinear discrete-time stochastic systems with interval timevarying delays and nonlinear disturbances. The nonlinear disturbances are more complex with uncertainty and timevarying delays. By constructing a new Lyapunov-Krasovskii functional and utilizing some well-known inequalities, we 
present novel delay-dependent criteria which guarantee the robust stochastic stability of a class of uncertain discrete-time stochastic systems. Then based on a state feedback control law, we give the delay-dependent sufficient conditions of robust stochastic stabilization for a class of uncertain discretetime stochastic systems with interval time-varying delays, and the controller gain is designed. In this paper, we convert the complex stability analysis problem into the resolvable LMI problem. The results of this paper can be easily extended to the global exponential stability problem.

\section{Conflict of Interests}

The authors declare that there is no conflict of interests regarding the publication of this paper

\section{References}

[1] J.-L. Wang and H.-N. Wu, "Robust stability and robust passivity of parabolic complex networks with parametric uncertainties and time-varying delays," Neurocomputing, vol. 87, pp. 26-32, 2012.

[2] Y. Guo, Y. Wang, and L. Xie, "Robust stability of reset control systems with uncertain output matrix," Automatica, vol. 48, no. 8, pp. 1879-1884, 2012.

[3] K. Ramakrishnan and G. Ray, "Robust stability criteria for a class of uncertain discrete-time systems with time-varying delay," Applied Mathematical Modelling, vol. 37, no. 3, pp. 14681479, 2013.

[4] H. Gao, J. Lam, and C. Wang, "Robust energy-to-peak filter design for stochastic time-delay systems," Systems \& Control Letters, vol. 55, no. 2, pp. 101-111, 2006.

[5] H. Huang, D. W. C. Ho, and J. Lam, "Stochastic stability analysis of fuzzy Hopfield neural networks with time-varying delays," IEEE Transactions on Circuits and Systems II: Express Briefs, vol. 52, no. 5, pp. 251-255, 2005.

[6] M. S. Mahmoud and N. B. Almutairi, "Robust stability and stabilization methods for a class of nonlinear discrete-time delay systems," Applied Mathematics and Computation, vol. 215, no. 12, pp. 4280-4292, 2010.

[7] S. Sathananthan, M. J. Knap, A. Strong, and L. H. Keel, "Robust stability and stabilization of a class of nonlinear discrete time stochastic systems: an LMI approach," Applied Mathematics and Computation, vol. 219, no. 4, pp. 1988-1997, 2012.

[8] Y. Liu and M. Li, "Improved robust stabilization method for linear systems with interval time-varying input delays by using Wirtinger inequality," ISA Transactions, vol. 56, pp. 111-122, 2015.

[9] Y. Dong, Y. Zhang, and X. Zhang, "Design of observer-based feedback control for a class of discrete-time nonlinear systems with time-delay," Applied and Computational Mathematics, vol. 13, no. 1, pp. 107-121, 2014.

[10] X. Mao, Stochastic Differential Equations with Markovian Switching, Imperial College Press, London, UK, 2006.

[11] Y. Zhang, D. Yue, and E. Tian, "Robust delay-distributiondependent stability of discrete-time stochastic neural networks with time-varying delay," Neurocomputing, vol. 72, no. 4-6, pp. 1265-1273, 2009.

[12] X. Wu, Y. Tang, and W. Zhang, "Stability analysis of switched stochastic neural networks with time-varying delays," Neural Networks, vol. 51, pp. 39-49, 2014.
[13] Y. Liu, Z. Wang, and X. Liu, "Robust stability of discretetime stochastic neural networks with time-varying delays," Neurocomputing, vol. 71, no. 4-6, pp. 823-833, 2008.

[14] J.-N. Li and L.-S. Li, "Mean-square exponential stability for stochastic discrete-time recurrent neural networks with mixed time delays," Neurocomputing, vol. 151, no. 2, pp. 790-797, 2015.

[15] J. Fu, H. Zhang, and T. Ma, "Delay-probability-distributiondependent robust stability analysis for stochastic neural networks with time-varying delay," Progress in Natural Science, vol. 19, pp. 1333-1340, 2009.

[16] T. L. Fernando, V. N. Phat, and H. M. Trinh, "Output feedback guaranteed cost control of uncertain linear discrete systems with interval time-varying delays," Applied Mathematical Modelling, vol. 37, no. 3, pp. 1580-1589, 2013.

[17] S. P. Bhattacharyya, H. Chapellat, and L. H. Keel, Robust Control: The Parametric Approach, Prentice-Hall, Upper Saddle River, NJ, USA, 1995.

[18] K. Zhou, J. C. Doyle, and K. Glover, Robust and Optimal Control, Prentice-Hall, Upper Saddle River, NJ, USA, 1996.

[19] E. K. Boukas, "State feedback stabilization of nonlinear discretetime systems with time-varying time delay," Nonlinear Analysis: Theory, Methods \& Applications, vol. 66, no. 6, pp. 1341-1350, 2007.

[20] J.-E. Feng and S. Xu, "Robust $H_{\infty}$ control with maximal decay rate for linear discrete-time stochastic systems," Journal of Mathematical Analysis and Applications, vol. 353, no. 1, pp. 460469, 2009.

[21] A. Cetinkaya and T. Hayakawa, "Feedback control of switched stochastic systems using randomly available active mode information," Automatica, vol. 52, pp. 55-62, 2015. 


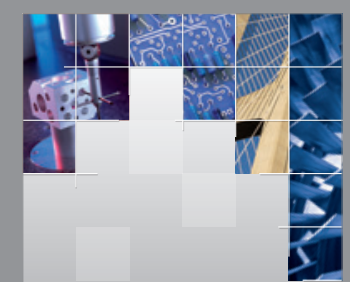

\section{Enfincering}
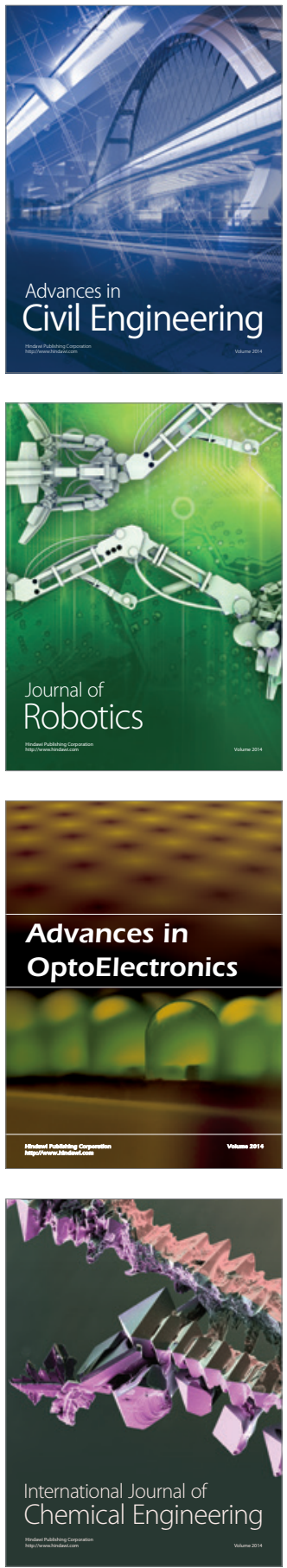

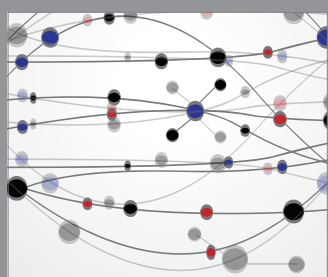

The Scientific World Journal

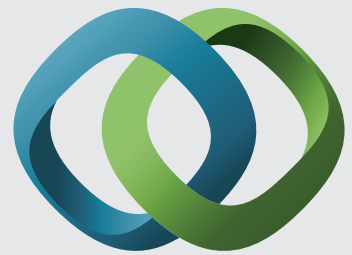

\section{Hindawi}

Submit your manuscripts at

http://www.hindawi.com
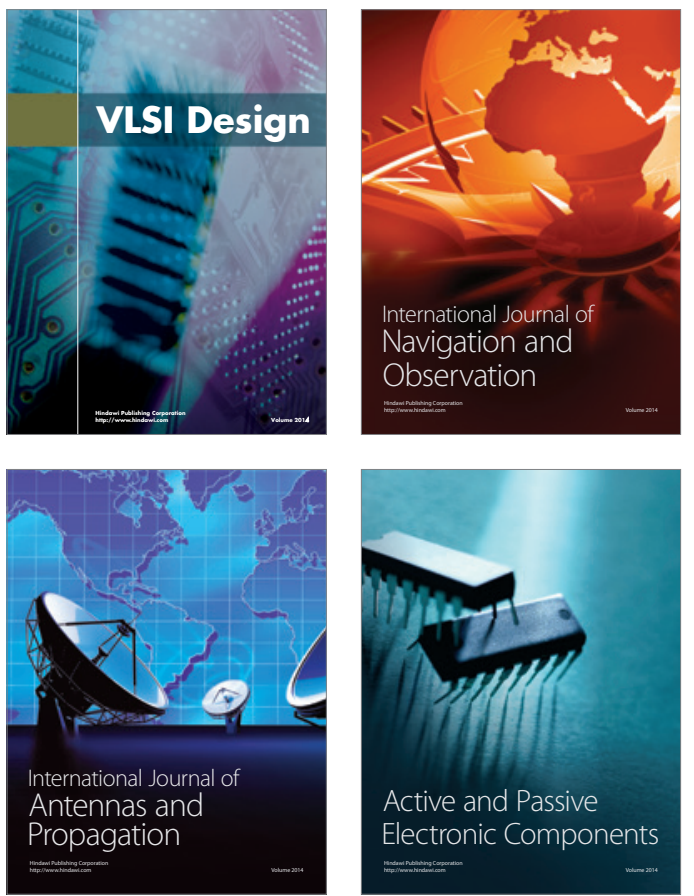
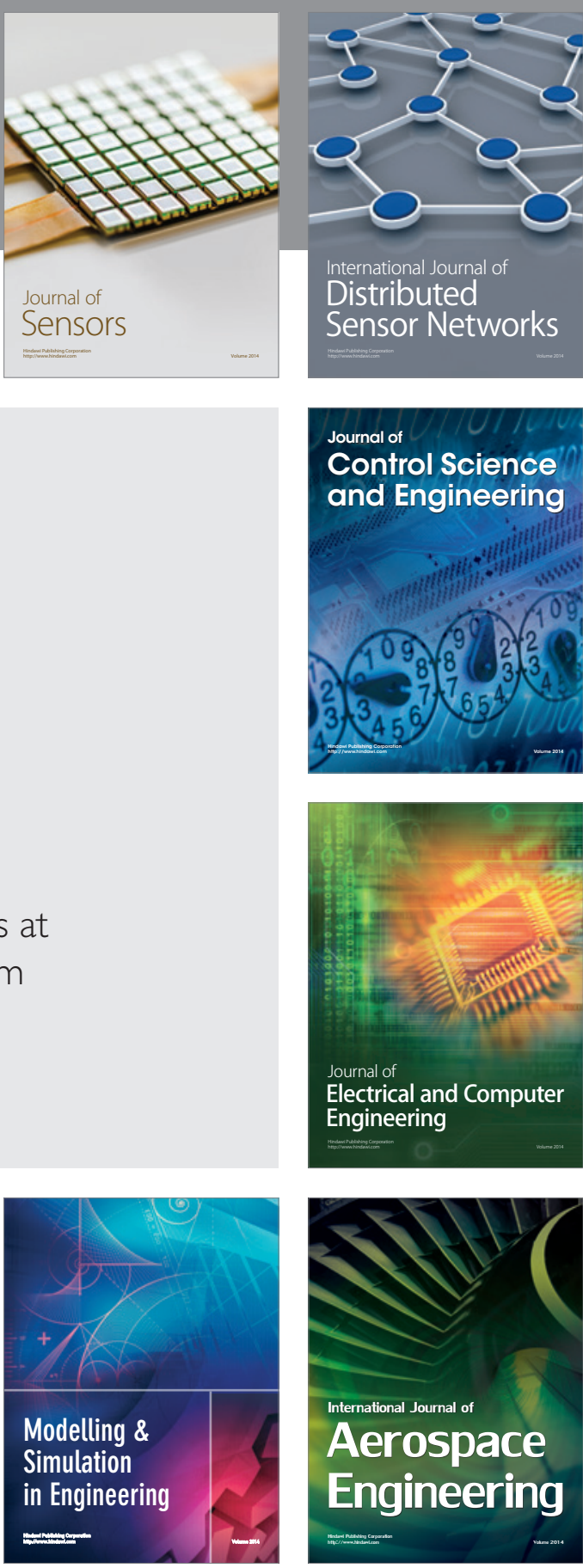

International Journal of

Distributed

Sensor Networks

Journal of

Control Science

and Engineering
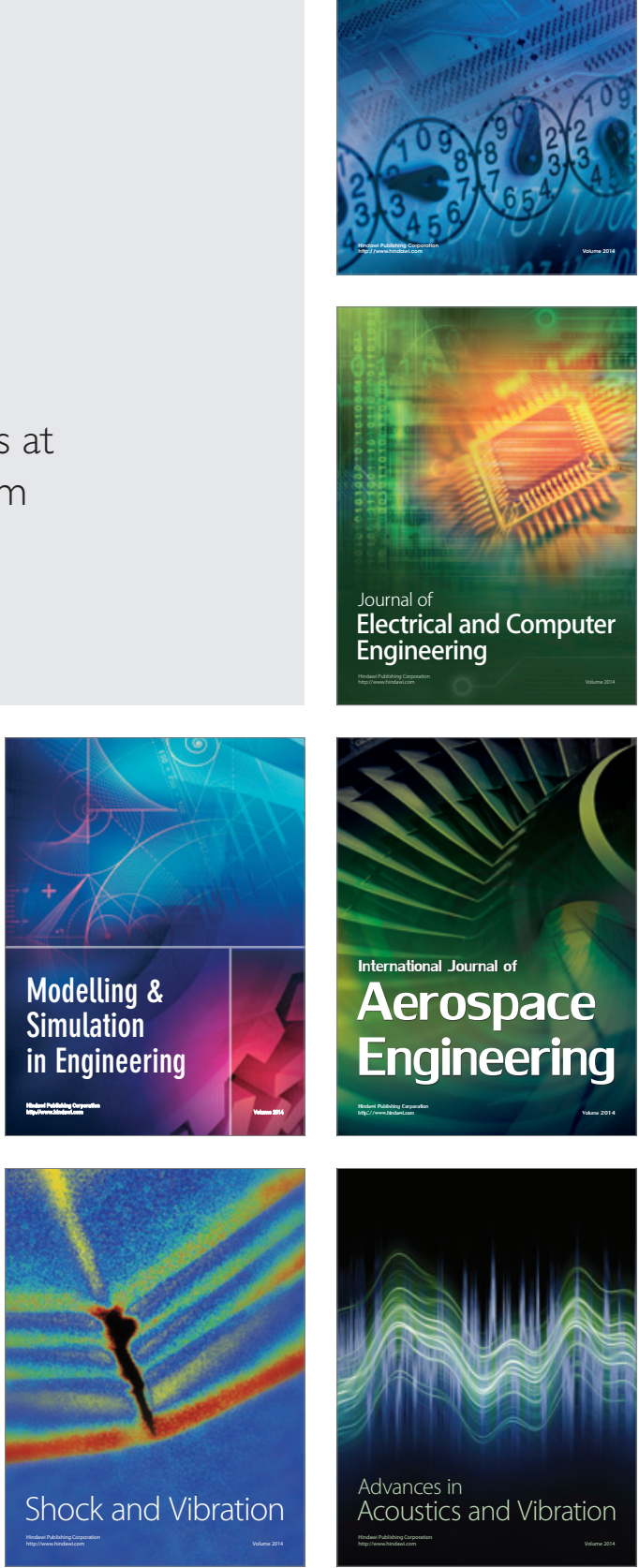